\title{
ON ASYMPTOTIC DISTRIBUTIONS OF ARITHMETICAL FUNCTIONS*
}

\author{
BY \\ I. J. SCHOENBERG
}

\section{INTRODUCTION}

1. The present note was suggested by recent work of $H$. Davenport, [3], $\dagger$ S. Bochner and B. Jessen, [2], and A. Wintner and B. Jessen, [6]. Davenport established the existence of asymptotic distribution functions for a certain class of arithmetical functions by an extension of a method previously used by the author, [8], [9], in a similar investigation. This method was based on the consideration of the moments of the distribution functions. In questions of asymptotic distribution, however, Bochner and Jessen have shown the great advantage of dealing directly with the Fourier transforms of the distribution functions. This advantage becomes again apparent if the method of Fourier transforms, whose adaptation to sequences is fully developed in §I, is applied to Davenport's problem. This is precisely what we shall do in §II; the result thus obtained (Theorem 1) insures the existence of the asymptotic distribution function for a very large class of (positive and multiplicative) arithmetical functions. It includes Davenport's and the author's previous results and yields readily (by suitable specializations of the arithmetical function involved) the frequencies of certain classes of integers investigated by W. Feller and E. Tornier, [4], in an entirely different way.

The connection with the work of Wintner and Jessen, [6], is as follows. The distribution function $\omega(x)=\chi\left(e^{u}\right)$ of Theorem 1 is a special example of the infinite convolutions of purely discontinuous distribution functions investigated by these authors. They have shown ([6], Theorem 35) that such infinite convolutions can be only either purely discontinuous or else everywhere continuous, and in the latter case either singular functions or else absolutely continuous functions. These general results apply immediately to our special situation, but new and probably difficult problems arise which may be mentioned here. Theorem 1 gives simple sufficient conditions to insure continuity or discontinuity of $\omega(x)$; the problem of finding similar necessary and sufficient conditions for continuity remains unsolved. The more delicate problem of deciding whether a continuous $\omega(x)$ is singular or absolutely

* Presented to the Society, March 31, 1934; received by the editors July 25, 1935.

$\dagger$ Numbers in brackets refer to the Bibliography at the end of this paper. 
continuous is likewise unsolved. It can be shown that a continuous $\omega(x)$ is necessarily singular if $\log f\left(p_{n}\right)=O\left(k^{-n}\right) \quad\left(p_{n}=n\right.$th prime, $\left.k>1\right)$. However, I do not have any example of an absolutely continuous $\omega(x)$.

\section{Asymptotic Distributions of REAL SEQUENCES}

2. Let us recall first a few well known definitions. A finite or infinite class $C$ of increasing positive integers $m_{1}, m_{2}, m_{3}, \cdots$ is said to have a frequency (or density) $F\{C\}$ if

$$
\lim _{n \rightarrow \infty}(1 / n) \sum_{m_{\nu} \leqq n} 1=F\{C\} .
$$

In case this limit does not exist then the upper limit of the same expression is the upper frequency $\bar{F}\{C\}$ and the lower limit is the lower frequency $\underline{F}\{C\}$ of the class $C$. A function $\omega(x)$ defined for $-\infty<x<\infty$, which is monotonic with $\omega(-\infty)=0, \omega(\infty)=1$, is called a distribution function (d.f.). A real sequence $x_{1}, x_{2}, x_{3}, \cdots$ is said to have an asymptotic distribution function (abbreviated: a.d.f.) $\omega(x)$ if for every point of continuity $x=\xi$ of $\omega(x)$ we have

$$
F\left\{x_{n} \leqq \xi\right\}=\omega(\xi) .
$$

In this relation $F\left\{x_{n} \leqq \xi\right\}$ means the frequency of the class of integers $n$ for which $x_{n} \leqq \xi$. For example we have so called equi-distribution* in the interval $(0,1)$ if the above definition holds with $\omega(x)=0$ for $x<0,=x$ for $0 \leqq x \leqq 1$, $=1$ for $x>1$.

Let $N\left(x_{\nu} \leqq x\right)_{n}$ denote the number of those elements among the first $n$ elements of the sequence $\left\{x_{\nu}\right\}$ which are $\leqq x$. With our sequence we may connect a sequence of distribution functions (step functions)

$$
\omega_{n}(x)=(1 / n) N\left(x_{\nu} \leqq x\right)_{n} \quad(-\infty<x<\infty ; n=1,2,3, \cdots) .
$$

A comparison with our previous definition shows that the d.f. $\omega(x)$ is the a.d.f. of our sequence $\left\{x_{n}\right\}$ if and only if the relation

$$
\lim _{n \rightarrow \infty} \omega_{n}(\xi)=\omega(\xi)
$$

holds for every continuity point $x=\xi$ of $\omega(x)$. The limiting relation (2) is usually described by saying that the sequence of d.f. $\omega_{n}(x)$ converges essentially to the d.f. $\omega(x)$.

Throughout this note we write

$$
\frac{g\left(x_{1}\right)+g\left(x_{2}\right)+\cdots+g\left(x_{n}\right)}{n}=M_{n}\{g(x)\}, \quad \lim _{n \rightarrow \infty} M_{n}\{g(x)\}=M\{g(x)\},
$$

${ }^{*}$ H. Weyl, [10]. 
provided the last limit exists. An important property of asymptotic distributions of sequences is contained in the following

Lemma 1. Let the sequence $\left\{x_{n}\right\}$ admit the a.d.f. $\omega(x)$ and let $g(x)$ be a bounded continuous complex-valued function for $-\infty<x<\infty$. Then

$$
M\{g(x)\}=\int_{-\infty}^{\infty} g(x) d \omega(x) .
$$

Since

$$
M_{n}\{g(x)\}=\int_{-\infty}^{\infty} g(x) d \omega_{n}(x),
$$

the relation (3) is a special case of a theorem of $P$. Lévy.*

3. The following theorem gives a criterion for asymptotic distributions of sequences.

LEMMA 2. Necessary and sufficient conditions that a sequence $\left\{x_{n}\right\}$ shall have an asymptotic distribution are as follows: The mean value

$$
M\left\{e^{i t x}\right\}=\lim _{n \rightarrow \infty} \frac{1}{n}\left(e^{i t x_{1}}+\cdots+e^{i t x_{n}}\right)=L(t)
$$

shall exist for every real $t$ and be an everywhere continuous function of $t$. If these conditions hold then $L(t)$ is of the form

$$
L(t)=\int_{-\infty}^{\infty} e^{i t x} d \omega(x)
$$

where $\omega(x)$ is the a.d.f. of our sequence $\left\{x_{n}\right\}$.

The necessity of these conditions is a consequence of Lemma 1 . In view of (4) we have

$$
\begin{aligned}
\sum_{\mu, \nu=1}^{m} L\left(t_{\mu}-t_{\nu}\right) \rho_{\mu} \bar{\rho}_{\nu} & =\sum_{\mu, \nu} M\left\{e^{i\left(t_{\mu}-t_{\nu}\right) x}\right\} \rho_{\mu} \bar{\rho}_{\nu}=M\left\{\sum_{\mu, \nu} e^{i\left(t_{\mu}-t_{\nu}\right) x} \rho_{\mu} \bar{\rho}_{\nu}\right\} \\
& =M\left\{\left|\sum_{\mu=1}^{m} e^{i t_{\mu} x} \rho_{\mu}\right|^{2}\right\} \geqq 0,
\end{aligned}
$$

hence $L(t)$ is a positive-definite function which, being assumed continuous,

*P. Lévy, [7], pp. 195-196. Lévy's theorem is as follows: If a sequence of d.f. $\omega_{n}(x)$ converges essentially to a d.f. $\omega(x)$, then

$$
\int_{-\infty}^{\infty} g(x) d \omega_{n}(x) \rightarrow \int_{-\infty}^{\infty} g(x) d \omega(x)
$$

for every bounded continuous $g(x)$. 
is of the form (5) with a non-decreasing $\omega(x)$ uniquely defined by $\omega(-\infty)=0$. For $t=0$ we get $\omega(+\infty)=L(0)=1$, hence $\omega(x)$ is a d.f. From (4) we infer

$$
\lim _{n \rightarrow \infty} \int_{-\infty}^{\infty} e^{i t x} d \omega_{n}(x)=\int_{-\infty}^{\infty} e^{i t x} d \omega(x) \text { for all real } t,
$$

and a theorem of P. Lévy† insures the relation (2), i.e., $\omega(x)$ is the a.d.f. of our sequence.

\section{Asymptotic Distributions OF MULTIPLICATIVE ARITHMETICAL FUNCTIONS}

4. Let $f(n)$ be a multiplicative arithmetical function, that is, a function defined for $n=1,2,3, \cdots$ and satisfying the relations

$$
f(m n)=f(m) f(n) \text { if }(m, n)=1, \quad f(1)=1 .
$$

As an immediate consequence of the unique factorization of integers into powers of primes, a multiplicative function is completely defined by prescribing arbitrarily the values of $f\left(p^{\alpha}\right)$ for all primes $p$ and integers $\alpha \geqq 1$. In describing such functions we therefore need to consider only the $f\left(p^{\alpha}\right)$.

Our problem is as follows: Under what assumptions does the sequence $u_{n}=f(n)$ have an asymptotic distribution function, and how is this function connected with the $f\left(p^{\alpha}\right)$ ? The results of this note in this direction are contained in the following

THEOREM 1. Let a multiplicative arithmetical function $f(n)$ satisfy the conditions

(i) $f\left(p^{\alpha}\right)>0$,

(ii) the series

* This follows from an important theorem of Bochner: A continuous positive-definite function is of the form (5) with a non-decreasing $\omega(x)$. The converse is obviously true. See Bochner [1], p. 76.

$\dagger$ In fact Lévy, [7], p. 197, in deriving (2) from (6), assumes that (6) holds uniformly in every finite $t$-interval. That this additional assumption is not necessary was shown by Bochner, [1], p. 72, Theorem 21. Bochner's statement proves that we can add suitable constants to our functions $\omega_{n}(x)$ so as to make them tend essentially to $\omega(x)$, i.e., there is a sequence of constants $c_{n}$ and a sequence of functions $\psi_{n}(x)$ such that

$$
\omega_{n}(x)+c_{n}=\omega(x)+\psi_{n}(x) \text { and } \lim _{n \rightarrow \infty} \psi_{n}(x)=0
$$

at every point of continuity of $\omega(x)$. From $c_{n}=\omega(x)-\omega_{n}(x)+\psi_{n}(x)$ we derive for every point of continuity of $\omega(x)$ the inequalities

$$
\omega(x)-1 \leqq \omega(x)-\lim \sup \omega_{n}(x) \leqq \lim \inf c_{n} \leqq \lim \sup c_{n} \leqq \omega(x)-\lim \inf \omega_{n}(x) \leqq \omega(x) ;
$$

allowing here $x \rightarrow \infty$ and $x \rightarrow-\infty$, we derive the result $\lim \inf c_{n}=\lim \sup c_{n}=0$, hence (2) holds. For our particular purpose (Theorem 1) Lévy's restricted statement would suffice, for without more trouble we can prove that (16) (the analogue of (4)) holds uniformly in every finite $t$-interval. 


$$
\sum_{p} \frac{1}{p}\|\log f(p)\| \text { converges, }
$$

where, as a matter of notation, $\|x\|=\min (1,|x|)$. Then $f(n)$ has the following properties:

1. The sequence $u_{n}=f(n)$ has an asymptotic distribution function $\chi(u)$ with

$$
\chi(u)=\chi(+0)=0 \text { for } u \leqq 0,
$$

and the Fourier transform of $\chi\left(e^{x}\right)(-\infty<x<\infty)$ is

$$
\begin{aligned}
\int_{-\infty}^{\infty} e^{i t x} d \chi\left(e^{x}\right)=L(t)= & \prod_{p}\left\{( 1 - \frac { 1 } { p } ) \left(1+\frac{1}{p} \exp [i t \log f(p)]\right.\right. \\
& \left.\left.+\frac{1}{p^{2}} \exp \left[i t \log f\left(p^{2}\right)\right]+\cdots\right)\right\},
\end{aligned}
$$

the infinite product being absolutely and uniformly convergent in every finite $t$ interval.

2. The set of points of increase of the distribution function $\chi(u)$ is identical with the sequence of points $u_{n}=f(n)$ together with the limit points of this sequence.

3. The distribution function $\chi(u)$ is purely discontinuous if the series

$$
\sum_{f(p) \neq 1} \frac{1}{p} \text { converges. }
$$

The function $\chi(u)$ is everywhere continuous if there exists a sequence of increasing primes, $q_{1}, q_{2}, q_{3}, \cdots$, with

$$
f\left(q_{\mu}\right) \neq f\left(q_{\nu}\right) \text { for } \mu \neq \nu,
$$

and such that

$$
\sum_{p=1}^{\infty} \frac{1}{q_{v}} \text { diverges.* }
$$

* Davenport's conditions (see [3], p. 10) for the existence of $\chi(u)$ are as follows:

(i') $0<f(n) \leqq 1$,

(ii') there are two positive constants $C$ and $c$ such that

$$
0 \leqq f\left(p^{\alpha-1}\right)-f\left(p^{\alpha}\right) \leqq C p^{-c \alpha}
$$

for $\alpha \geqq 1$ and all primes $p$.

One should remark first that Theorem 1 imposes no conditions whatever on the values of $f\left(p^{\alpha}\right)$ for $\alpha>1$, except condition (i). Moreover, for $\alpha=1$ (ii') gives $0 \leqq 1-f(p) \leqq C p^{-c}$ and this implies already the convergence of our series (8). Hence all of the inequalities $\left(8^{\prime}\right)$ for $\alpha>1$ are superfluous as far as the existence of the d.f. $\chi(u)$ is concerned. 
5. In order to prove the first part of Theorem 1 let us consider the infinite product

$$
\begin{aligned}
L(t)= & \prod_{p}\left\{1+p^{-1}(\exp [i t \log f(p)]-1)\right. \\
& \left.+p^{-2}\left(\exp \left[i t \log f\left(p^{2}\right)\right]-\exp [i t \log f(p)]\right)+\cdots\right\}=\prod_{p}\left(1+a_{p}\right) .
\end{aligned}
$$

By means of the inequalities

$$
|\sin x| \leqq\|x\|=\min (1,|x|), \quad\|x t\| \leqq\|x\| \max (1,|t|),
$$

we have

$$
\begin{aligned}
\left|a_{p}\right| \leqq & p^{-1}|\exp [i t \log f(p)]-1| \\
& +p^{-2}\left|\exp \left[i t \log f\left(p^{2}\right)\right]-\exp [i t \log f(p)]\right|+\cdots \\
\leqq & 2 p^{-1}\left|\sin \frac{t \log f(p)}{2}\right|+2\left(p^{-2}+p^{-3}+\cdots\right) \\
\leqq & 2 p^{-1}\left\|\frac{t}{2} \log f(p)\right\|+2 p^{-1}(p-1)^{-1} \\
< & 2 p^{-1}\|\log f(p)\| \max \left(1, \frac{|t|}{2}\right)+2(p-1)^{-2} \\
= & p^{-1} \| \log f\left(p^{2} \| \max (2,|t|)+2(p-1)^{-2} .\right.
\end{aligned}
$$

For $|t| \leqq T$, the series $\sum a_{p}$ is therefore dominated by the convergent series

$$
\sum_{p}\left(p^{-1}\|\log f(p)\| \max (2, T)+2(p-1)^{-2}\right)
$$

and the infinite product (13) converges absolutely and uniformly in every finite $t$-interval. As a further consequence of the last result we have

$$
\prod_{p}\left\{1+\sum_{\alpha=1}^{\infty} p^{-\alpha}\left|\exp \left[i t \log f\left(p^{\alpha}\right)\right]-\exp \left[i t \log f\left(p^{\alpha-1}\right)\right]\right|\right\}=\sum_{m=1}^{\infty} \frac{\left|\rho_{t}(m)\right|}{m}
$$

with

$$
\begin{aligned}
\rho_{t}(m) & =\prod_{p^{\alpha} \mid m}\left(\exp \left[i t \log f\left(p^{\alpha}\right)\right]-\exp \left[i t \log f\left(p^{\alpha-1}\right)\right]\right) \\
& =\sum_{d \mid m} \mu(d) \exp \left[i t \log f\left(\frac{m}{d}\right)\right],
\end{aligned}
$$

where $p^{\alpha}$ are the powers of different primes in the canonical decomposition of $m$ and where $\mu(m)$ is the Möbius function. From the convergence of the last series one readily derives the relation 


$$
\lim _{n \rightarrow \infty} \frac{1}{n} \sum_{m=1}^{n}\left|\rho_{t}(m)\right|=0 .
$$

By the inversion formula of Möbius, (14) implies

$$
\exp [i t \log f(m)]=\sum_{d \mid m} \rho_{t}(d),
$$

and therefore

$$
\begin{aligned}
\frac{1}{n} \sum_{m=1}^{n} \exp [i t \log f(m)] & =\frac{1}{n} \sum_{m=1}^{n} \sum_{d \mid m} \rho_{t}(d)=\frac{1}{n} \sum_{m=1}^{n}\left[\frac{n}{m}\right] \rho_{t}(m) \\
& =\sum_{m=1}^{\infty} \frac{\rho_{t}(m)}{m}+\frac{1}{n} \sum_{m=1}^{n}\left\{\frac{n}{m}-R\left(\frac{n}{m}\right)\right\} \rho_{t}(m)-\sum_{m=1}^{\infty} \frac{\rho_{t}(m)}{m} \\
& =\sum_{m=1}^{\infty} \frac{\rho_{t}(m)}{m}-\frac{1}{n} \sum_{m=1}^{n} R\left(\frac{n}{m}\right) \rho_{t}(m)-\sum_{m=n+1}^{\infty} \frac{\rho_{t}(m)}{m},
\end{aligned}
$$

where $R(x)=x-[x]$. Using (15) we derive

$$
\lim _{n \rightarrow \infty} \frac{1}{n} \sum_{m=1}^{n} e^{i t \log f(m)}=\sum_{m=1}^{\infty} \frac{\rho_{t}(m)}{m}=L(t),
$$

where $L(t)$ is the infinite product (13). Since $L(t)$ is continuous, (16) and Lemma 2 show that the sequence $\{\log f(n)\}$ admits an a.d.f. $\omega(x)$ whose Fourier transform is (5), hence the original sequence $\{f(n)\}$ admits the a.d.f. $\chi(u)$ defined as follows:

$$
\chi(u)=\left\{\begin{array}{cc}
0 & \text { for } u \leqq 0, \\
\omega(\log u) & \text { for } u>0,
\end{array}\right.
$$

with $\chi(+0)=\omega(-\infty)=0$. The remark that $\omega(x)=\chi\left(e^{x}\right)$ completes the proof of the first part of Theorem 1.

6. Let us pass to the proof of the second part of Theorem 1. Jessen and Wintner ([6], Theorem 3) have proved the following general result. Let $\sigma_{1}(x), \sigma_{2}(x), \sigma_{3}(x), \cdots$ be a sequence of d.f. such that the convolution $\omega_{n}(x)=\sigma_{1}(x) * \sigma_{2}(x) * \cdots * \sigma_{n}(x)$ converges essentially to a d.f. $\omega(x)$; this d.f. $\omega(x)$ is called the infinite convolution of the sequence $\left\{\sigma_{\nu}(x)\right\}$, and we write

$$
\omega(x)=\sigma_{1}(x) * \sigma_{2}(x) * \sigma_{3}(x) * \cdots .
$$

Let generally $S(\phi)$ denote the set of points of increase of a d.f. $\phi$. Then $S\left(\omega_{n}\right)$ is the vectorial sum of sets (in the sense of Bohr)

$$
S\left(\omega_{n}\right)=S\left(\sigma_{1}\right)+S\left(\sigma_{2}\right)+\cdots+S\left(\sigma_{n}\right),
$$


and $S(\omega)$ is the limit of $S\left(\omega_{n}\right)$ in the following sense: A point $\xi$ belongs to $S(\omega)$ if and only if it is the limit of a sequence of points $x_{n}$ with $x_{n} \subset S\left(\omega_{n}\right)$.

If the origin $O$ belongs to all $S\left(\sigma_{n}\right)$, then $S\left(\omega_{1}\right) \subset S\left(\omega_{2}\right) \subset S\left(\omega_{3}\right) \subset \cdots$ in virtue of (18), and now $S(\omega)$ is identical with the closure of the ordinary limit of $S\left(\omega_{n}\right)$, i.e., a point belongs to $S(\omega)$ if it belongs to some $S\left(\omega_{n}\right)$ or else is a limit of such points. Formula (9) shows that our d.f. $\omega(x)=\chi\left(e^{x}\right)$ is the infinite convolution of the sequence of d.f. $\sigma_{\nu}(x)$ of Fourier transforms

$$
\begin{aligned}
\int_{-\infty}^{\infty} e^{i t x} d \sigma_{v}(x)= & \left(1-p_{\nu}^{-1}\right)\left(1+p_{\nu}^{-1} \exp \left[i t \log f\left(p_{v}\right)\right]\right. \\
& \left.+p_{v}^{-2} \exp \left[i t \log f\left(p_{v}^{2}\right)\right]+\cdots\right)
\end{aligned}
$$

where $p_{\nu}$ stands for the $\nu$ th prime; hence $S\left(\omega_{n}\right)$ is identical with the set of points $\log f\left(p_{1}{ }^{\alpha_{1}} p_{2}{ }^{\alpha_{2}} \cdots p_{n}{ }^{\alpha_{n}}\right)\left(\alpha_{\nu} \geqq 0\right)$ and the second part of Theorem 1 is established.

7. Passing to the third part of Theorem 1 we remark that (10) implies (8). However, if (10) converges we need not consider (8) at all, for now the series

$$
\sum_{p} p^{-1}(\exp [i t \log f(p)]-1)
$$

is dominated by the convergent series

$$
\sum_{f(p) \neq 1} 2 p^{-1}
$$

for all values of $t$, which implies the uniform convergence of product (13) for all real $t$. The transform $L(t)$ is therefore almost periodic and the d.f. $\omega(x)$ is necessarily purely discontinuous.

Let us now assume (8), (11) and (12) to hold. By (17) and (19) we have

$$
\omega=\sigma_{1} * \sigma_{2} * \sigma_{3} * \cdots=\left(\sigma_{1} * \cdots * \sigma_{n-1} * \sigma_{n+1} * \cdots\right) * \sigma_{n}=\phi_{n} * \sigma_{n} .
$$

Denoting by $\tilde{\omega}(E), \tilde{\phi}_{n}(E), \tilde{\sigma}_{n}(E)$ the set functions corresponding to the point functions $\omega, \phi_{n}, \sigma_{n}$, we have

$$
\tilde{\omega}(E)=\int \tilde{\phi}_{n}(E-y) \tilde{\sigma}_{n}(d y) \cdot \dagger
$$

If $\omega(x)$ is not everywhere continuous, there is a point $x$ with $\tilde{\omega}(x)=c>0$. Now by (19), writing $\log f\left(p_{n}\right)=\lambda_{n}$,

$$
\begin{aligned}
\tilde{\omega}(x) & =\int \tilde{\phi}_{n}(x-y) \tilde{\sigma}_{n}(d y) \\
& =\tilde{\phi}_{n}(x)\left(1-\frac{1}{p_{n}}\right)+\tilde{\phi}_{n}\left(x-\lambda_{n}\right)\left(\frac{1}{p_{n}}-\frac{1}{p_{n}^{2}}\right)+\cdots=c>0 .
\end{aligned}
$$

† A Lebesgue-Radon integral. For notation and references see [6], \$2. 
Since $0 \leqq \tilde{\phi}_{n}(E) \leqq 1$, we may infer that

$$
\tilde{\phi}_{n}(x)>c / 2 \text { for } n \text { sufficiently large, }
$$

hence

$$
\begin{aligned}
\tilde{\omega}\left(x+\lambda_{n}\right) & =\tilde{\phi}_{n}\left(x+\lambda_{n}\right)\left(1-\frac{1}{p_{n}}\right)+\tilde{\phi}_{n}(x)\left(\frac{1}{p_{n}}-\frac{1}{p_{n}^{2}}\right)+\cdots \\
& >\frac{c}{2}\left(\frac{1}{p_{n}}-\frac{1}{p_{n}^{2}}\right) .
\end{aligned}
$$

This result, however, leads to a contradiction, for $\sum \tilde{\omega}\left(x+\lambda_{n}\right)$, summed over all different $\lambda_{n}$ (with $n$ large), is on one hand $\leqq 1$, on the other hand it is $>(c / 2) \sum\left(p_{n}^{-1}-p_{n}^{-2}\right)$, and this last series diverges by our assumptions (11) and (12).*

\section{Frequencies of CERTAin Classes of INTEgers}

8. Theorem 1 applies with great ease to the multiplicative arithmetical functions

$$
\begin{array}{ll}
f(n)=\phi(n) / n & (\phi(n) \text { is the Euler function }), \dagger \\
f(n)=n / \sigma(n) & (\sigma(n)=\text { sum of divisors of } n), \ddagger
\end{array}
$$

for $f(p)=1-1 / p$ and $p /(1+p)$ respectively, and both series

$$
\sum_{p} \frac{1}{p}\left|\log \left(1-\frac{1}{p}\right)\right|, \quad \sum_{p} \frac{1}{p} \log \left(1+\frac{1}{p}\right)
$$

are convergent. Moreover both functions are everywhere dense in the interval $(0,1)$, as even their values of the form

$$
f\left(q_{1} q_{2} \cdots q_{m}\right)=\left(1-\frac{1}{q_{1}}\right) \cdots\left(1-\frac{1}{q_{m}}\right) \text { and } \frac{q_{1}}{1+q_{1}} \cdots \frac{q_{m}}{1+q_{m}} \text { respectively, }
$$

where $q_{1}, \cdots, q_{m}$ are different primes, have this property. $\S$ Since the $f(p)$ are all different, the distribution functions are continuous. We can therefore conclude that the frequency $F\{\sigma(n)>k n\}$ of $k$-abundant numbers $\|$ is continuous and strictly decreasing for increasing $k \geqq 1$.

* The conditions (11), (12), and the above proof of their sufficiency are due to Dr. Jessen. My original conditions were more stringent.

† Schoenberg [8].

$\ddagger$ Davenport [3].

$\S$ See [8], p. 194.

|| Davenport [3], p. 830. 
These results may be extended to various generalizations of Euler's function as well as to the function

$$
f(n)=n^{s} / \sigma_{s}(n)=n^{s} / \sum_{d \mid n} d^{s}
$$

For $0<s \leqq 1$, this last function is everywhere dense in $(0,1)$; if $s>1$ we have

$$
\lim \inf f(n)=1 / \zeta(s), \quad \lim \sup f(n)=1 . \dagger
$$

9. We now shall apply Theorem 1 to certain arithmetical functions leading to purely discontinuous distribution functions. A few preliminary considerations are necessary.

Let $x_{1}, x_{2}, x_{3}, \cdots$ be a sequence of real elements. Let $\lambda_{1}, \lambda_{2}, \lambda_{3}, \cdots$ be all the different values of the elements of the sequence $\left\{x_{n}\right\}$, i.e., $\lambda_{\mu} \neq \lambda_{\nu}$ if $\mu \neq \nu$ while any $x_{n}$ is equal to some $\lambda_{\mu}$ and vice versa. Let us further assume that the sequence $\left\{x_{n}\right\}$ has an a.d.f. $\omega(x)$ whose Fourier transform is

$$
\int_{-\infty}^{\infty} e^{i t x} d \omega(x)=\sum_{m=1}^{\infty} A_{m}^{i t \lambda_{m}}
$$

hence

$$
A_{m} \geqq 0, \quad \sum_{m=1}^{\infty} A_{m}=1 .
$$

We shall need the following

LEMma 3. If the sequence $\left\{\lambda_{\mu}\right\}$ has no finite limit point, then

$$
\begin{aligned}
& F\left\{x_{\nu}=\lambda_{m}\right\}=A_{m}, \\
& F\left\{x_{\nu}=\lambda_{m_{1}}, \lambda_{m_{2}}, \lambda_{m_{3}}, \cdots\right\}=A_{m_{1}}+A_{m_{2}}+A_{m_{3}}+\cdots \\
&\left(m_{1}<m_{2}<m_{3}<\cdots\right) .
\end{aligned}
$$

For if the interval $\lambda_{m}-\epsilon \cdots \lambda_{m}+\epsilon$ is free of values $\lambda_{\mu}(\mu \neq m)$, then $\lambda_{m} \pm \epsilon$ are points of continuity (in fact points of constancy) of $\omega(x)$, hence

$$
\lim _{n \rightarrow \infty} \frac{1}{n} N\left(x_{\nu} \leqq \lambda_{m} \pm \epsilon\right)_{n}=\omega\left(\lambda_{m} \pm \epsilon\right)=\omega\left(\lambda_{m} \pm 0\right)
$$

and therefore

$$
\begin{aligned}
\lim _{n \rightarrow \infty} \frac{1}{n}\left\{N\left(x_{\nu} \leqq \lambda_{m}+\epsilon\right)_{n}-N\left(x_{\nu} \leqq \lambda_{m}-\epsilon\right)_{n}\right\} & =\lim _{n \rightarrow \infty} \frac{1}{n} N\left(x_{\nu}=\lambda_{m}\right)_{n} \\
& =\omega\left(\lambda_{m}+0\right)-\omega\left(\lambda_{m}-0\right)=A_{m},
\end{aligned}
$$

$\dagger$ Gronwall [5]; presumably $f(n)$ is everywhere dense in $\left(\zeta^{-1}(s), 1\right)$. 
Let $C_{m}$ be the class of integers $\nu$ for which $x_{\nu}=\lambda_{m}$. The totality $T$ of all positive integers is thus decomposed as a sum of classes

$$
T=C_{1}+C_{2}+C_{3}+\cdots \text {, with } F\left\{C_{m}\right\}=A_{m},
$$

and (23) is equivalent to

$$
F\left\{C_{m_{1}}+C_{m_{2}}+\cdots\right\}=F\left\{C_{m_{1}}\right\}+F\left\{C_{m_{2}}\right\}+\cdots \cdot \dagger
$$

From

we get

$$
C=C_{m_{1}}+C_{m_{2}}+\cdots+C_{m_{k-1}}+\left(C_{m_{k}}+C_{m_{k+1}}+\cdots\right)
$$

$$
\bar{F}\{C\} \leqq F\left\{C_{m_{1}}\right\}+\cdots+F\left\{C_{m_{k-1}}\right\}+\bar{F}\left\{C_{m_{k}}+C_{m_{k+1}}+\cdots\right\} .
$$

On the other hand

$$
\begin{aligned}
\bar{F}\left\{C_{m_{k}}+C_{m_{k+1}}+\cdots\right\} & \leqq \bar{F}\left\{C_{m_{k}}+C_{m_{k}+1}+C_{m_{k}+2}+\cdots\right\} \\
& =1-F\left\{C_{1}+C_{2}+\cdots+C_{m_{k}-1}\right\} \\
& =1-F\left\{C_{1}\right\}-\cdots-F\left\{C_{m_{k}-1}\right\} \rightarrow 0 \text { as } k \rightarrow \infty
\end{aligned}
$$

in view of (21). Hence, as $k \rightarrow \infty,(25)$ gives

$$
\bar{F}\{C\} \leqq F\left\{C_{m_{1}}\right\}+F\left\{C_{m_{2}}\right\}+\cdots \cdot
$$

This together with the obvious relation

$$
\underline{F}\{C\} \geqq F\left\{C_{m_{1}}\right\}+F\left\{C_{m_{2}}\right\}+\cdots
$$

proves (24).

10. Let us devide the totality $T$ of positive integers into various classes as follows. Call $C(1)$ the class of square-free numbers. If $n$ is not square-free let $q_{1}{ }^{\alpha_{1}} q_{2}{ }^{\alpha_{2}} \ldots q_{r}^{\alpha_{r}}\left(\alpha_{1}>1, \cdots, \alpha_{r}>1\right)$ be the product of all the powers of primes in its canonical decomposition and call $C\left(q_{1}{ }^{\alpha_{1}} q_{2}{ }^{\alpha_{2}} \ldots q_{r}^{\alpha_{r}}\right)$ the class of all numbers $n$ having the same product $q_{1}^{\alpha_{1}} \ldots q_{r}^{\alpha_{r}}$ of powers of primes in their canonical decomposition.

What is the frequency $F\left\{C\left(q_{1}^{\alpha_{1}} q_{2}^{\alpha_{2}} \cdots q_{r}^{\alpha_{r}}\right)\right\}$ of the class $C\left(q_{1}^{\alpha_{1}} q_{2}{ }^{\alpha_{2}} \cdots q_{r}^{\alpha_{r}}\right)$ ? It can be immediately computed from (9) by specializing conveniently the function $f(n)$. For all primes $p$ let

$$
f(p)=1, f\left(p^{\alpha}\right)=p^{\alpha} \text { for } \alpha>1 .
$$

The series (10) is void and the a.d.f. $\omega(x)$ of the sequence $\{\log f(n)\}$ is purely discontinuous. Since $\Pi\left(1-p^{-2}\right)=\zeta(2)^{-1}=6 / \pi^{2}$, its Fourier transform given by (9) becomes

$\dagger$ This relation, which I owe to Dr. Jessen, is not true for every decomposition $T=C_{1}+C_{2}+\cdots$, for even the relation $F\left\{C_{1}+C_{2}+\cdots\right\}=F\left\{C_{1}\right\}+F\left\{C_{2}\right\}+\cdots$ breaks down in the case of the decomposition $T=(1)+(2)+(3)+\cdots$. 


$$
\begin{array}{r}
\int_{-\infty}^{\infty} e^{i t x} d \omega(x)=L(t)=\frac{6}{\pi^{2}} \prod_{p}\left\{1+p^{-2}\left(1+\frac{1}{p}\right)^{-1} \exp \left[i t \log p^{2}\right]\right. \\
\left.+p^{-3}\left(1+\frac{1}{p}\right)^{-1} \exp \left[i t \log p^{3}\right]+\cdots\right\} \\
=\frac{6}{\pi^{2}}\left\{1+\sum_{\left(q_{1}^{\alpha_{1}} \cdots q_{r}^{\alpha_{r}}\right)}^{(\alpha>1)} q_{1}^{-\alpha_{1}} q_{2}^{-\alpha_{2}} \cdots q_{r}^{-\alpha_{r}}\left(1+\frac{1}{q_{1}}\right)^{-1}\right. \\
\left.\ldots\left(1+\frac{1}{q_{r}}\right)^{-1} \exp \left[i t \log \left(q_{1}^{\alpha_{1}} \cdots q_{r}^{\alpha_{r}}\right)\right]\right\} .
\end{array}
$$

Since $\log f(n)=\log \left(q_{1}{ }^{\alpha_{1}} q_{2}{ }^{\alpha}{ }_{2} \ldots q_{r}{ }^{\alpha r}\right)(\alpha>1)$ if and only if $n$ belongs to the class $C\left(q_{1}{ }^{\alpha_{1}} q_{2}^{\alpha_{2}} \ldots q_{r}^{\alpha_{r}}\right)$ and since the various values of $\log \left(q_{1}{ }^{\alpha_{1}} \ldots q_{r}^{\alpha_{r}}\right)$ have no finite limit point (as logarithms of different integers) Lemma 3 shows that

$$
\begin{aligned}
& F\left\{C\left(q_{1}^{\alpha_{1}} q_{2}^{\alpha_{2}} \cdots q_{r}^{\alpha_{r}}\right)\right\} \\
& \quad=\frac{6}{\pi^{2}} q_{1}^{-\alpha_{1}} q_{2}^{-\alpha_{2}} \cdots q_{r}^{-\alpha_{r}}\left(1+\frac{1}{q_{1}}\right)^{-1} \cdots\left(1+\frac{1}{q_{r}}\right)^{-1} .
\end{aligned}
$$

By the same lemma we obtain the frequency of a sum of classes $C\left(q_{1}{ }^{\alpha_{1}} \ldots q_{r}{ }^{\alpha_{r}}\right)$ by simply adding the frequencies of the individual classes. $\dagger$ Feller and Tornier determine the frequency of the class of numbers $n$ which have an even number of powers of primes in their canonical decomposition (loc. cit., p. 229). We may obtain their result directly from (28), for

$$
\begin{aligned}
F\left\{\sum_{r \text { even }} C\left(q_{1}^{\alpha_{1}} \cdots q_{r}^{\alpha_{r}}\right)\right\} & =\frac{6}{\pi^{2}} \sum_{r \text { even }} \frac{1}{\left(q_{1}^{2}-1\right) \cdots\left(q_{r}^{2}-1\right)} \\
& =\frac{6}{2 \pi^{2}}\left\{\prod_{p}\left(1+\frac{1}{p^{2}-1}\right)+\prod_{p}\left(1-\frac{1}{p^{2}-1}\right)\right\} \\
& =\frac{1}{2} \prod_{p}\left(1-p^{-2}\right)\left\{\prod_{p} \frac{1}{1-p^{-2}}+\prod_{p} \frac{1-2 p^{-2}}{1-p^{-2}}\right\} \\
& =\frac{1}{2}+\frac{1}{2} \prod_{p}\left(1-2 p^{-2}\right) .
\end{aligned}
$$

11. The derivation of (28) was essentially based on the fact that the sequence $\{\log f(n)\}$ defined by (26) has an a.d.f. $\omega(x)$ with the transform (27). It is of some interest to point out that this result may be derived by means of elementary properties of trigonometric polynomials only, without reference to Stieltjes integrals or the more refined theorems used in $\$ 1$. To discuss a 
somewhat more general situation than (26) let us assume (a) that the series (10) converges, (b) that the sequence $\left\{\lambda_{m}\right\}$ of the different values of the elements of the sequence $\{\log f(n)\}$ has $+\infty$ as its only limiting point, in which case by renumbering the $\lambda_{m}$ we may assume $\lambda_{1}<\lambda_{2}<\lambda_{3}<\cdots<\lambda_{m} \rightarrow \infty$.

We know that (10) implies that $L(t)$ is an almost periodic function with a Fourier expansion of the type

$$
L(t)=\sum_{m=1}^{\infty} A_{m} e^{i\left(\lambda \lambda_{m}\right.} \quad\left(A_{m} \geqq 0, \sum_{1}^{\infty} A_{m}=1\right) .
$$

Another immediate consequence of (10) is that the limiting relation (16) holds uniformly for all real $t$. Hence if we write

$$
\frac{1}{n} \sum_{m=1}^{n} e^{i t \log f(m)}=\sum^{*} a_{m}^{(n)} e^{i\left(\lambda_{m}\right.}
$$

we know that these polynomials converge uniformly to $L(t)$. From $A_{m}=\mathfrak{M}_{t}\left\{L(t) e^{-i t \lambda_{m}}\right\}$ and the similar formulas for our polynomials we get

$$
\lim _{n \rightarrow \infty} a_{m}^{(n)}=A_{m} \quad(m=1,2,3, \cdots),
$$

and therefore (in the notation of $\$ 1$ )

$$
\omega_{n}(x)=\sum_{\lambda_{m}<x} a_{m}^{(n)} \rightarrow \sum_{\lambda_{m}<x} A_{m}=\omega(x)
$$

for every $x \neq \lambda_{1}, \lambda_{2}, \cdots$, for both of the sums involved in the last relation contain a finite number of terms only. But this relation means precisely that $\omega(x)$ is the a.d.f. of the sequence $\{\log f(n)\}$.

12. A great number of examples of classes of integers could be indicated whose frequencies can be computed by the method used above. We shall discuss only two more examples already considered by Feller and Tornier ([4], pp. 215 and 224).

Let $\Gamma$ be the class of numbers of the form $n=q_{1}{ }^{\alpha_{1}} q_{2}{ }^{\alpha_{2}} \cdots q_{r}^{\alpha_{r}}(\alpha>1)$, i.e., if $p \mid n$ then also $p^{2} \mid n$. To compute $F\{\Gamma\}$ let $\Gamma_{m}$ be the class of numbers for which the above property $\left(p \mid n\right.$ implies $\left.p^{2} \mid n\right)$ is required only for the first $m$ primes $p_{1}, p_{2}, \cdots, p_{m}$. Obviously $\Gamma \subset \Gamma_{m}$. Consider the multiplicative funćtion $f(n)$ defined by

$$
\begin{gathered}
f\left(p_{1}\right)=p_{1}, f\left(p_{2}\right)=p_{2}, \cdots, f\left(p_{m}\right)=p_{m}, f\left(p_{m+1}\right)=f\left(p_{m+2}\right)=\cdots=1, \\
f\left(p^{\alpha}\right)=1 \text { for } \alpha>1 .
\end{gathered}
$$

(10) is fulfilled and (9) becomes 


$$
L(t)=\prod_{v=1}^{m}\left\{1-p_{\nu}^{-1}+p_{\nu}^{-2}+p_{\nu}^{-1}\left(1-p_{\nu}^{-1}\right) \exp \left[i t \log p_{v}\right]\right\} .
$$

Since $\log f(n)=0$ if and only if $n \subset \Gamma_{m},(22)$ gives

$$
\bar{F}\{\Gamma\} \leqq F\left\{\Gamma_{m}\right\}=\prod_{\nu=1}^{m}\left(1-p_{\nu}^{-1}+p_{\nu}^{-2}\right) \text { which } \rightarrow 0 \text { as } m \rightarrow \infty,
$$

hence $F\{\Gamma\}=0$.

Let $k_{1}, k_{2}, k_{3}, \cdots$ be a sequence of positive integers some of which may be infinite and let $K$ be the class of integers not divisible by any of the prime powers $p_{1}^{k_{1}}, p_{2}^{k_{2}}, p_{3}^{k_{3}}, \cdots\left(k_{m}=\infty\right.$ means that there is no restriction at all with respect to $p_{m}$ ). To determine $F\{K\}$ let us assume first that the series

$$
\sum_{\nu=1}^{\infty} p_{\nu}^{-k}
$$

converges. Define a multiplicative function $f(n)$ by

$$
\begin{aligned}
f\left(p_{v}\right)=f\left(p_{\nu}^{2}\right)=\cdots=f\left(p_{v}^{k_{v}-1}\right)=1, f\left(p_{\nu}^{\alpha}\right)=p_{\nu}^{\alpha} & \text { for } \alpha \geqq k_{\nu} \\
& (\nu=1,2,3, \cdots) .
\end{aligned}
$$

Since

$$
\sum_{f(p) \neq 1} p^{-1}=\sum_{\left(k_{\nu}=1\right)} p_{\nu}^{-1} \leqq \sum_{\nu=1}^{\infty} p_{\nu}^{-k_{\nu}}
$$

converges, the a.d.f. of $\{\log f(n)\}$ is a step function with the transform

$$
\begin{aligned}
L(t)=\prod_{\nu=1}^{\infty}\left\{( 1 - p _ { \nu } ^ { - 1 } ) \left(1+p_{v}^{-1}+\cdots\right.\right. & +p_{\nu}^{-\left(k_{\nu}-1\right)} \\
& \left.\left.+p_{\nu}^{-k_{\nu}} \exp \left[i t \log p_{\nu} k_{\nu}\right]+\cdots\right)\right\} .
\end{aligned}
$$

Since $\log f(n)=0$ if and only if $n \subset K$, we have

(31) $F\{K\}=\prod_{\nu=1}^{\infty}\left\{\left(1-p_{\nu}^{-1}\right)\left(1+p_{\nu}^{-1}+\cdots+p_{\nu}^{-\left(k_{\nu}-1\right)}\right)\right\}=\prod_{\nu=1}^{\infty}\left(1-p_{\nu}^{-k_{\nu}}\right)$.

If (30) diverges let $K_{m}$ be the class of integers similar to $K$ but for the new sequence of exponents $k_{1}, k_{2}, \cdots, k_{m}, \infty, \infty, \cdots$; then $K \subset K_{m}$ and (31) applied for the new sequence gives

$$
\bar{F}\{K\} \leqq F\left\{K_{m}\right\}=\prod_{\nu=1}^{m}\left(1-p_{\nu}^{-k_{\nu}}\right) \text { which } \rightarrow 0 \text { as } m \rightarrow \infty .
$$

Hence $F\{K\}=0$ and the formula (31) is again valid.

Obviously our last two examples are also particular cases of the elementary scheme discussed above. 
Added in proof, January, 1936. In a recent note On the density of some sequences of numbers, Journal of the London Mathematical Society, vol. 10 (1935), pp. 120-125, P. Erdös proves without using Fourier analysis two theorems which in our notation are as follows.

1. If $f(n)$ is a multiplicative function satisfying the conditions

$$
\begin{aligned}
& f(n) \geqq 1, \\
& \sum_{p}\|\log f(p)\| / p \text { converges, } \\
& f\left(p_{1}\right) \neq f\left(p_{2}\right) \text { if } p_{1}, p_{2} \text { are different primes, }
\end{aligned}
$$

the sequence $\{f(n)\}$ has a continuous asymptotic distribution function.

2. If the multiplicative function $f(n)$ satisfying (32) is such that

$$
\sum_{p}\|\log f(p)\| / p \text { diverges, }
$$

then

$$
F\{f(n) \geqq \tau\}=1 \text { for any real } \tau \geqq 1 \text {. }
$$

The first theorem of Erdös is obviously a consequence of Theorem 1. This is not true for the second theorem; I want to show, however, how it can be derived from Theorem 1 by a simple additional argument involving moments rather than Fourier transforms.

Let $f_{k}(n)$ be an auxiliary multiplicative function defined as follows:

$$
\begin{aligned}
& f_{k}\left(p_{\nu}^{\alpha}\right)=f\left(p_{\nu}^{\alpha}\right) \quad(\nu=1,2, \cdots, k), \\
& f_{k}\left(p_{\nu}^{\alpha}\right)=1 \quad(\nu=k+1, k+2, \cdots ; \alpha \geqq 1) .
\end{aligned}
$$

The sequence $\left\{\log f_{k}(n)\right\}$ has an a.d.f. $\omega_{k}(x)$ and the a.d.f. of the sequence $\left\{f_{k}^{-1}(n)\right\}$ (contained within $0<t \leqq 1$ ) is therefore $\chi_{k}(t)=\omega_{k}(-\log t)$. For $s>0$ we have

$$
\begin{aligned}
& \prod_{v=1}^{k}\left\{\left(1-p_{\nu}^{-1}\right)\left(1+p_{\nu}^{-1} \exp \left[-s \log f\left(p_{\nu}\right)\right]+\cdots\right)\right\} \\
& =\int_{0}^{\infty} e^{-s x} d \omega_{k}(x)=\int_{0}^{1} t^{s} d \chi_{k}(t) .
\end{aligned}
$$

The product (38) tends to zero as $k \rightarrow \infty$ on account of (35). But

$$
\lim _{k \rightarrow \infty} \int_{0}^{1} t^{s} d \chi_{k}(t)=0, \text { for } s>0,
$$

implies (see [8], pp. 175-176) $\chi_{k}(t) \rightarrow 1$ for $0<t \leqq 1$. Hence 


$$
\begin{aligned}
E\{f(n) \geqq \tau\} & =\underline{F}\left\{f^{-1}(n) \leqq \tau^{-1}\right\} \geqq F\left\{f_{k}^{-1}(n) \leqq \tau^{-1}\right\} \\
& =\chi_{k}\left(\tau^{-1}\right) \rightarrow 1 \text { as } k \rightarrow \infty
\end{aligned}
$$

and (36) is proved. The last inequality for frequencies follows from $f^{-1}(n) \leqq f_{k}^{-1}(n)$ which is due to (32) and (37).

\section{BIBLIOGRAPHY}

1. S. Bochner, Vorlesungen über Fouriersche Integrale, Leipzig, 1932.

2. S. Bochner and B. Jessen, Distribution functions and positive-definite functions, Annals of Mathematics, (2), vol. 35 (1934), pp. 252-257.

3. H. Davenport, Über numeri abundantes, Sitzungsberichte der Preussischen Akademie, Physikalisch-Mathematische Klasse, 1933, pp. 830-837.

4. W. Feller und E. Tornier, Mengentheoretische Untersuchungen von Eigenschaften der Zahlenreihe, Mathematische Annalen, vol. 107 (1932), pp. 188-232.

5. T. H. Gronwall, Some asymptotic expressions in the theory of numbers, these Transactions, vol. 14 (1913), pp. 113-122.

8. B. Jessen and A. Wintner, Distribution functions and the Riemann zeta function, these Transactions, vol. 38 (1935), pp. 48-88.

7. P. Lévy, Calcul des Probabilités, Paris, 1925.

8. I. J. Schoenberg, Über die asymptotische Verteilung reeller Zahlen mod 1, Mathematische Zeitschrift, vol. 28 (1928), pp. 171-200.

9. I. J. Schoenberg, Über total monotone Folgen mit stetiger Belegungsfunktion, vol. 30 (1929), pp. 761-767.

10. H. Weyl, Über die Gleichverteilung von Zahlen mod 1, Mathematische Annalen, vol. 77 (1916), pp. 313-352.

Institute for Advanced Study,

Princeton, N. J., AND

SWARTHMORE COLLEge,

Swarthmore, Pa. 